\title{
Stress Relief as the Driving Force for Self-Assembled Bi Nanolines
}

\author{
J. H. G. Owen and K. Miki* \\ Nanotechnology Research Institute (NRI), National Institute of Advanced Industrial Science and Technology (AIST), \\ AIST Tsukuba Central 4, Higashi 1-1-1, Tsukuba, Ibaraki 305-8562, Japan \\ and Nanomaterials Laboratory, National Institute of Materials Science (NIMS), Sengen 1-2-1, \\ Tsukuba, Ibaraki 305-0047, Japan \\ H. Koh and H.W. Yeom \\ Atomic-scale Surface Science Research Center and Institute of Physics and Applied Physics, Yonsei University, \\ Seoul 120-749, Korea \\ D. R. Bowler ${ }^{\dagger}$ \\ Department of Physics and Astronomy, University College London, Gower Street, London, WC1E 6BT, United Kingdom
} (Received 8 January 2002; published 17 May 2002)

\begin{abstract}
Bi nanolines self-assemble on $\mathrm{Si}(001)$ and are remarkable for their straightness and length - they are often more than $400 \mathrm{~nm}$ long, and a kink in a nanoline has never been observed. Through electronic structure calculations, we have found an energetically favorable structure for these nanolines that agrees with our scanning tunneling microscopy and photoemission experiments; the structure has an extremely unusual subsurface structure, comprising a double core of seven-membered rings of silicon. Our proposed structure explains all the observed features of the nanolines, and shows that surface stress resulting from the mismatch between the Bi and the Si substrates is responsible for their self-assembly. This has wider implications for the controlled growth of nanostructures on semiconductor surfaces.
\end{abstract}

DOI: 10.1103/PhysRevLett.88.226104

PACS numbers: 68.65.-k, 81.07.-b, 81.16.-c

Nanowires are of enormous importance for nanoelectronics: recently, various devices have been constructed from semiconductor nanowires [1] and carbon nanotubes [2], to name but two. These approaches require assembly on the surface, whether by fluidics and patterning or use of scanning probes; self-assembled nanowires would be a compelling alternative for fabricating a large number of devices. However, the microscopic understanding of the underlying physical and chemical mechanisms for self-assembly of nanoscale features has been limited. Surface stress resulting from lattice mismatch in heteroepitaxial growth has often been thought to be responsible for nanoscale roughness [3], and self-assembly of nanoscale features (such as the growth of Ge "hut" clusters [4]). For the case of self-assembled nanowires on semiconductor surfaces, there has been much recent work on rare-earth silicides (e.g., $\mathrm{ErSi}_{2}$ ), where there is a large strain along one axis $(\sim 7 \%)$ and almost none along another, leading to the formation of extended nanowires [5]. These wires, however, are far from being uniform or perfect at the atomic scale. In contrast, Bi nanolines, formed when a Bi-covered $\mathrm{Si}(001)$ surface is annealed at around $570-600{ }^{\circ} \mathrm{C}[6,7]$, are quite striking in their uniformity. These nanolines are always $1.5 \mathrm{~nm}$ wide, and extend for hundreds of nanometers without a kink or a defect. As well as this, they repel defects and down $B$-type step edges to a distance of 3-4 nm. Further, they are resistant to attack by radical hydrogen or oxygen (hence hydrogen can be used as a mask and oxygen used to isolate them electrically from the substrate) [8], making them promising as templates for nanowires of other materials. However, the structure of these Bi nanolines, a prerequisite to a microscopic understanding of their unique properties and hence control of their nucleation and growth, remains unknown.

Earlier, we proposed a structure based upon a threedimer wide model [6,9]. However, recent atomic resolution scanning tunneling microscopy (STM) images of the Bi nanoline $[8,10]$ have revealed that the structure, in fact, occupies the space of four dimers in the $\mathrm{Si}(001)$ surface. A proposed four-dimer model [10] has the wrong spacing of features in the nanoline (ca. 5.4 $\mathrm{A}$ ), and is energetically very poor (more than $0.6 \mathrm{eV} / \mathrm{Bi}$ dimer worse than the three-dimer model [9]). Moreover, neither model has a large kinking energy. Accordingly, we have conducted an exhaustive search for a new structure and tested many tens of candidates against experimental criteria, which we detail below. For this purpose, semiempirical tight binding (tb) calculations are invaluable, as they allow us to run large simulations of hundreds of atoms quickly on modest hardware (a desktop computer), while achieving relaxed energies very close to those obtained from ab initio density functional theory (DFT) calculations $[9,11]$. In this paper, we propose a new structure for these nanolines that is energetically favorable and agrees with all experimental observations (size, stability, registry with Si dimers, straightness, repulsion of defects). We note that it is related to a structure recently proposed for $B$-type steps on As-terminated $\mathrm{Ge}(001)$ surfaces, and that our findings may well have implications for group $\mathrm{V}$ elements on group IV surfaces in general. 
The $\mathrm{Si}(001)$ substrate was cleaned using a standard process [12] before being transferred into vacuum. The Si surface was prepared by flashing repeatedly to $1100{ }^{\circ} \mathrm{C}$ for a few seconds, until there was only a small pressure rise. The clean surface was checked with STM before Bi deposition began. Bi was evaporated from an effusion cell, a typical dose being $\mathrm{Bi}$ at $470{ }^{\circ} \mathrm{C}$ for $10 \mathrm{~min}$. STM images were taken at the deposition temperature between $570-600{ }^{\circ} \mathrm{C}$, and at room temperature, using a JEOL 4500 XT UHV STM. The high-resolution Bi $5 d$ core-level photoemission spectra, taken at $65 \mathrm{eV}$ photon energy, were measured on the new high-resolution vacuum ultraviolet beam line BL-1C at Photon Factory, KEK, Japan. The overall energy resolution was better than $100 \mathrm{meV}$. The relative stabilities of the proposed structures were calculated using two electronic structure techniques: for a swift search of possible motifs, tight binding; for accurate energies and structures, DFT. The tight binding calculations were performed with a linear scaling code using a parametrization which has been previously validated for $\mathrm{Bi}-\mathrm{Si}$ interactions [9]; this allowed the large unit cells necessary to model the long range strain effects seen in our STM data. The DFT calculations were performed using the VASP code [13], using ultrasoft pseudopotentials, a plane wave cutoff of $150 \mathrm{eV}$ (sufficient for energy difference convergence) and a Monkhurst-Pack k-point mesh with $4 \times 4 \times 1$ points. The unit cell used had ten layers of $\mathrm{Si}$, with 16 atoms in each layer [forming a single dimer row with the $p(2 \times 2)$ reconstruction] with the bottom two layers constrained to remain fixed and dangling bonds terminated in hydrogen. When comparing energies with different amounts of $\mathrm{Bi}$, we use unit cells of the same surface area, and compare the excess surface energy plus bismuth adsorption energy per Bi dimer [9].

The Bi nanolines have several notable and unusual features. Two features are their straightness and perfection. Hundreds of lines have been observed, without a kink being seen, and defects are extremely rare (their straightness can be seen in Fig. 1 and also in previous work [6,7]). This would suggest that the nanoline has a large kinking energy. Another feature is the "defect exclusion zone" (DEZ). Low concentrations of Bi embedded in the top layer of $\mathrm{Si}(001)$ cause compressive surface stress, and ordered trenches of missing dimer defects form every 8-12 dimers to relieve the stress. Despite being highly mobile at high temperatures, these defects do not come within 3-4 nm of the nanolines - the DEZ. Since the strain field of a missing dimer defect is tensile, the repulsive interaction between the defect and the nanolines suggests that the nanoline strain field should also be tensile, and hence is also a source of stress relief for the embedded Bi. It is likely therefore that the stress induced by the $\mathrm{Bi}$ in the top layer of the $\mathrm{Si}$ is the driving force for the formation of this unusual structure. Having formed, the nanolines remain after epitaxial islands of $\mathrm{Bi}$ have evaporated, indicating increased stability (RHEED experiments found the difference in desorption barrier to be $0.25 \mathrm{eV}$ [6]). How-

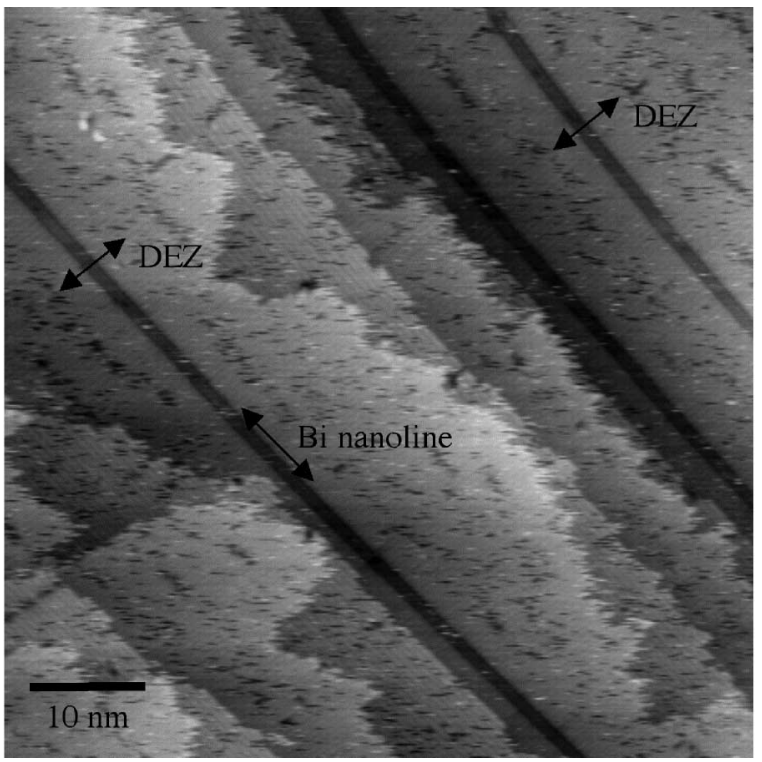

FIG. 1. A $65 \mathrm{~nm} \times 65 \mathrm{~nm}$ STM image of the Si(001) surface taken at $590{ }^{\circ} \mathrm{C}$, showing three $\mathrm{Bi}$ nanolines. The black streaks on the surface are rapidly moving defects. Around each nanoline, there is an area free of defects marked by the arrows as "DEZ" (defect exclusion zone). At this sample bias, the nanolines appear dark; at higher biases, the nanoline is bright relative to the silicon.

ever, the local structure of the $\mathrm{Bi}$ in the nanoline appears to be in a simple dimerized form, in the top surface layer. Recent high resolution STM images, such as in Fig. 2(a), and previous work $[8,10]$ show that the nanolines are four dimers or $15.4 \AA$ wide. The bright dimerlike features making up the nanoline lie between the underlying Si dimers, in line with the Si dimer rows. As marked, the spacing between the nanoline features is $6.3 \AA$. Photoemission spectroscopy (PES) experiments find that the Bi $5 d$ core-level spectra of the $\mathrm{Bi}$ nanowire is essentially identical to the spectra of the $(2 \times n)$ phase composed of Bi ad-dimers, with a single well defined spin-orbit doublet. This strongly suggests that the local chemical state and registry of $\mathrm{Bi}$ adsorbates for both phases are the same, i.e., that the $\mathrm{Bi}$ is in the form of dimers in the top layer of the structure. $\mathrm{X}$-ray photoelectron diffraction (XPD) experiments [14] confirm the presence of Bi dimers parallel to the Si dimers and find the spacing between them to be $\sim 6.3 \AA$ A. Hence, the observed properties of the Bi nanoline must result not from a novel $\mathrm{Bi}$ structure, such as a square of $\mathrm{Bi}$ atoms, but from an unusual $\mathrm{Si}$ substructure, stabilized by the presence of $\mathrm{Bi}$.

A simple model for the line, with two adjacent $\mathrm{Bi}$ ad-dimers sitting on top of the dimer row, as shown in Fig. 3(a), reproduces most of the aspects of the detailed STM data, and is consistent with the PES and XPD data. However, this structure is $0.53 \mathrm{eV} / \mathrm{Bi}$ dimer less stable than the $(2 \times n)$ phase, and has no energetic reason to grow long and straight; nor, indeed, is there any reason for the two ad-dimers to remain adjacent. However, 


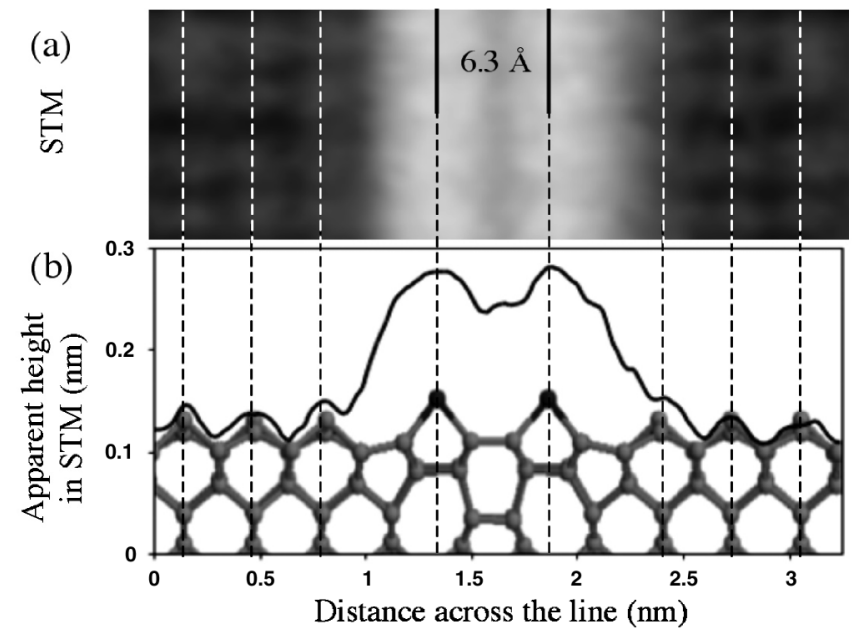

FIG. 2. (a) A Bi nanoline (on a H-terminated surface). The feature spacing within the nanoline is $6.3 \AA$. (H-termination of the background $\mathrm{Si}$ dimers makes them easier to resolve, so that the registry of the line relative to the surface can be confirmed.) (b) The side view of our proposed structure has been matched up to the STM cross section. Dotted lines mark the peak positions, showing that the model and the STM match extremely well.

our proposed structure may be reached from this simple structure by rearrangement of only a few atoms, illustrated in Fig. 3. A two-dimer wide core (equivalent to half of our proposed structure) can be formed by rotation of the 2nd/3rd layer atoms, so that they lie on the same level. The resulting structure is quite similar to that proposed for the structure of As-Ge double-height $B$-type steps [15]. The energy of this structure is lower than the simple ad-dimer model, but the strain field of the core is compressive, the wrong sign for stress relief, and two such cores close together, necessary to form a four-dimer unit, as shown in Fig. 3(b), repel each other. Removal of four central atoms and rebonding of 1st- and 4th-layer $\mathrm{Si}$ atoms makes the overall strain field tensile, while keeping all bonds saturated. This gives our proposed structure, shown in Fig. 3(c).

Our proposed structure is energetically very favorable and gives good agreement with all aspects of our experimental findings. In DFT calculations, the energy/Bi dimer is $-10.9 \mathrm{eV} / \mathrm{Bi}$ dimer, $0.25 \mathrm{eV} / \mathrm{Bi}$ dimer lower than the high coverage $\mathrm{Bi}-(2 \times n)$ phase. This energy difference agrees very well with the difference in stability as measured by RHEED. A cross section of the nanoline matched up with the ball-and-stick model is shown in Fig. 2(b). The position of the peaks of the Bi dimers line up perfectly with the STM profile. The spacing found from our calculations $(6.25 \AA)$ and the direction of the Bi dimers (parallel to Si dimers) agree extremely well with photoemission spectroscopy and with XPD results [14]. Since most of our candidate structures contain two Bi dimers in a four-dimer unit cell, as strongly suggested by STM, XPD, and PES data, the chemical potential diagram, shown in Fig. 4, is mostly composed of parallel lines. One excep-

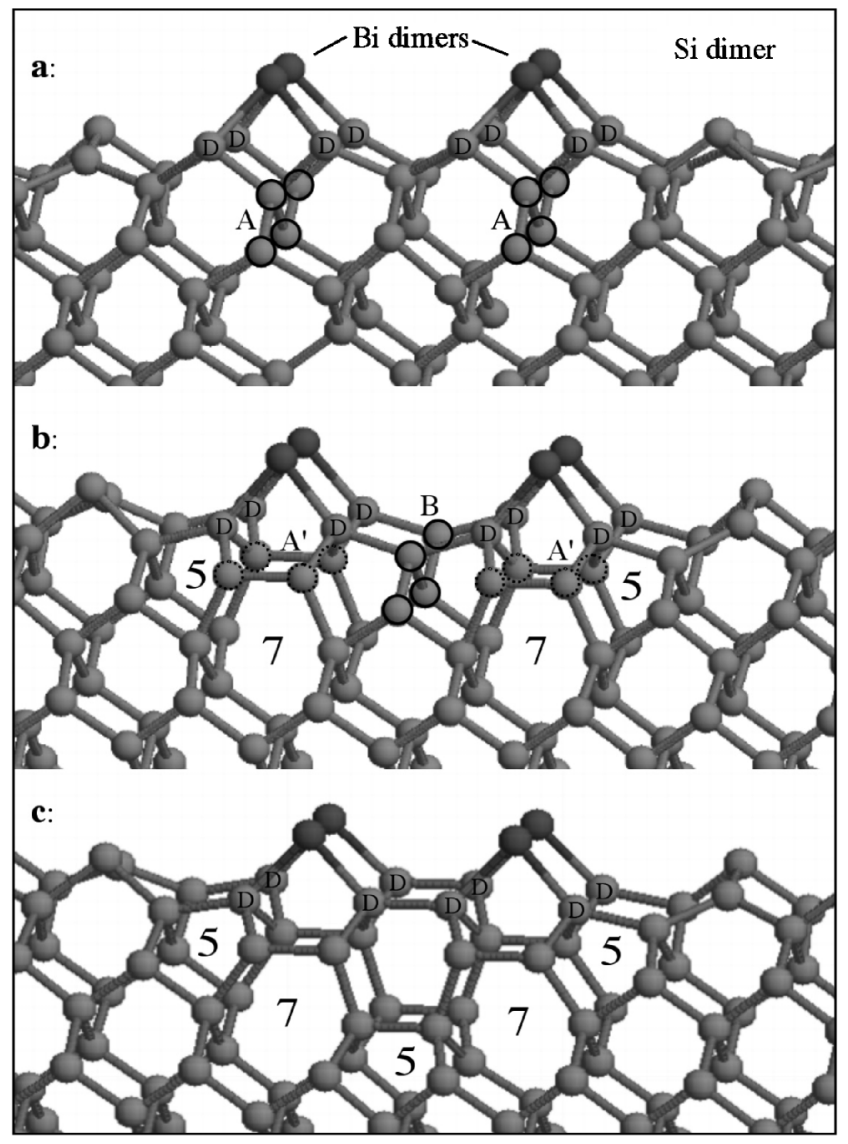

FIG. 3. Candidate Bi nanoline structures. (a) A simple ad-dimer structure is shown. The atoms marked " $D$ " are the original $\mathrm{Si}$ dimer atoms. Rotation of the $2 \mathrm{nd} / 3 \mathrm{rd}$ layer atoms (marked $\mathrm{A}$ ) beneath the $\mathrm{Bi}$ dimers, produces the second structure (b), with two cores of five- and seven-membered rings of $\mathrm{Si}$. This structure is under compressive stress, which may be relieved by the removal of the central four 2 nd/3rd layer atoms (marked $B$ ) and rebonding, resulting in our proposed structure (c). Our proposed structure has an energy $0.25 \mathrm{eV} /$ dimer better than the high coverage $\mathrm{Bi}-(2 \times n)$ surface.

tion is the line for the high coverage $\operatorname{Bi}-(2 \times n)$ phase, which crosses the line for our proposed structure around $-10.5 \mathrm{eV}$. However, these data are not reliable in this region, as at this potential, the compact islands are breaking up into isolated ad-dimers.

In conclusion, we have conducted an exhaustive search for a stable structure for the Bi nanoline, which satisfied all our constraints. The majority of our candidate structures contained two Bi dimers per unit cell, as is strongly suggested by the STM and PES data, but some structures involving squares of $\mathrm{Bi}$ atoms were also investigated. Unsuccessful candidates were rejected for a variety of reasons: energetically poor, the wrong appearance in STM, low kinking energy, or the lack of a significant strain field. These will be discussed in more detail in a future paper [11]. Not only is our proposed structure much more stable (ca. $0.5 \mathrm{eV} / \mathrm{Bi}$ dimer) than any other we have calculated and is more stable than the high coverage $\mathrm{Bi}-(2 \times n)$ 


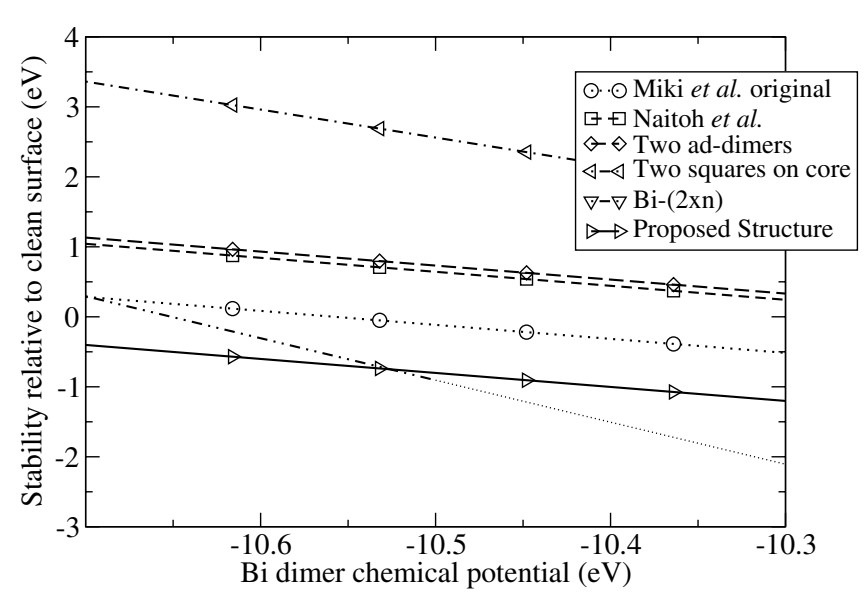

FIG. 4. A comparison of the energy per Bi dimer of various candidate structures across a range of $\mathrm{Bi}$ chemical potentials. The graph limits represent high (left) and low (right) Bi coverages. The Bi- $(2 \times n)$ line is continued in a dotted line beyond $\mu=-10.5 \mathrm{eV}$ as it is not valid in this region.

surface over a wide range of $\mathrm{Bi}$ chemical potential, it matches extremely well with criteria drawn from a variety of experimental data. Moreover, our proposed structure stands out from others that we have tested, in that it explains the other observed features of the Bi nanolines very naturally. The importance of surface strain relief in the formation of this structure is underlined by its increased stability in a surface terminated by Bi dimers. In this case, the total energy of a long tight-binding unit cell was further lowered by $\sim 0.1 \mathrm{eV} /$ dimer, due to relaxation of the compressive stress in the surface Bi dimers. The deep reconstruction leads to a large kinking energy [3.75 eV/kink (tb)], as expected, and the tensile strain field of the nanoline also provides a driving force for the DEZ. Tight-binding calculations on a 32-dimer cell found that single missing dimer defects and step edges interact repulsively with the nanoline out to a range of $\sim 3 \mathrm{~nm}$, in agreement with the observed DEZ width of 3-4 nm. We plan to report detailed calculations on all these features of the nanolines in future work. The large tensile strain in one direction and zero strain in the other is very similar to the situation seen in the silicide nanowires [5], and accounts in part for the invariant width and extreme length of the nanowire. Also of note are the five-membered and seven-membered rings of Si, marked in Figs. 3(b) and 3(c). Such oddmembered rings are also present in the proposed As-Ge step structure, suggesting that this structural motif may be of general interest in structures involving group $\mathrm{V}$ layers on group IV surfaces, particularly in situations where there is either compressive or tensile stress. Finally, the properties of these nanolines demonstrate the importance of surface stress in the formation and phenomenonology of nanoscale structures.

The authors thank Professor Andrew Briggs (Oxford University) and Bill McMahon for useful discussions, and Dr. Masaru Shimomura and Professor Shozo Kono (Tohoku University) for detailed discussions about their XPD experiments. D.R. B. acknowledges support from the EPSRC and from the Royal Society, and the HiPerSPACE Centre (UCL) for computer time for DFT calculations. J. H. G. O. is supported by the Japanese Science and Technology Agency (JST). H. K. and H. W. Y. are supported by KOSEF through ASSRC, BK 21 program, and Tera-level Nanodevice projects of 21 century Frontier program. This study was performed through Special Coordination Funds of the Ministry of Education, Culture, Sports, Science and Technology (MEXT) of the Japanese Government (Research Project on active atom-wire interconnects).

*Email address: miki.kazushi@aist.go.jp

${ }^{\dagger}$ Email address: david.bowler@ucl.ac.uk

[1] Y. Huang, X. Duan, Y. Cui, L. J. Lauhon, K.-H. Kim, and C. M. Lieber, Science 294, 1313 (2001).

[2] A. Bachtold, P. Hadley, T. Nakanishi, and C. Dekker, Science 294, 1317 (2001).

[3] L. J. Gray, M. F. Chisholm, and T. Kaplan, Appl. Phys. Lett. 66, 1924 (1995).

[4] D. J. Eaglesham and M. Cerullo, Phys. Rev. Lett. 64, 1943 (1990).

[5] Y. Chen, D. A. A. Ohlberg, G. Medeiros-Ribeiro, Y. A. Chang, and R.S. Williams, Appl. Phys. Lett. 76, 4004 (2000).

[6] K. Miki, J. H. G. Owen, D. R. Bowler, G. A. D. Briggs, and K. Sakamoto, Surf. Sci. 421, 397 (1999).

[7] K. Miki, D. R. Bowler, J. H. G. Owen, G. A. D. Briggs, and K. Sakamoto, Phys. Rev. B 59, 14868 (1999).

[8] J. H. G. Owen, D. R. Bowler, and K. Miki, Surf. Sci. Lett. 499, L124 (2002).

[9] D. R. Bowler, Phys. Rev. B 62, 7237 (2000).

[10] M. Naitoh, M. Takei, S. Nishigaki, N. Oishi, and F. Shoji, Jpn. J. Appl. Phys. Pt. 1 39, 2793 (2000).

[11] D. R. Bowler and J. H. G. Owen, cond-mat/0202530.

[12] K. Miki, K. Sakamoto, and T. Sakamoto, Surf. Sci. 406, 312 (1998).

[13] G. Kresse and J. Furthmüller, Comput. Mater. Sci. 6, 15 (1996).

[14] M. Shimomura, K. Miki, T. Abukawa, and S. Kono, Surf. Sci. Lett. 447, L169 (2000).

[15] S. B. Zhang, W. E. McMahon, J. M. Olson, and S.-H. Wei, Phys. Rev. Lett. 87, 166104 (2001). 